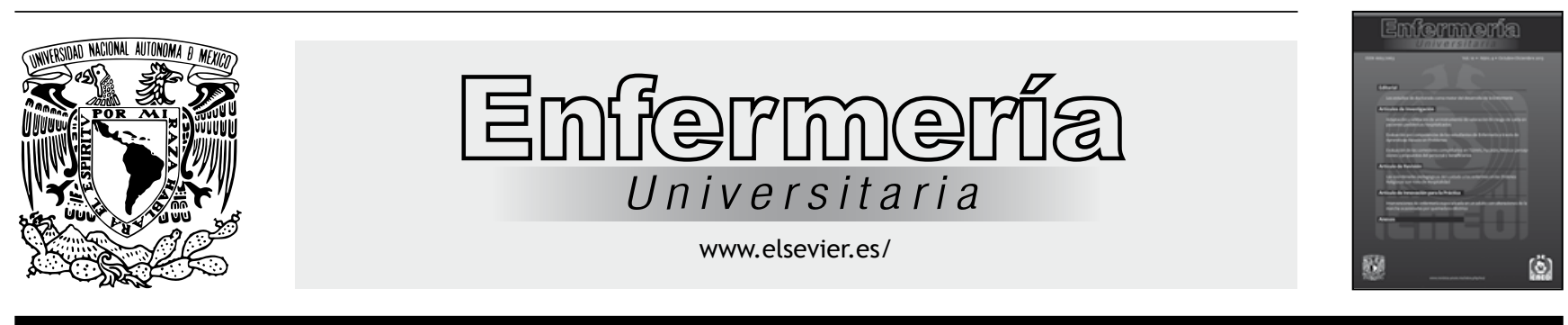

\title{
ÍNDICE ACUMULATIVO
}

\section{Revista no. 1 Año 10}

\section{Editorial}

Mirando al pasado

Mtra. Ma. D. Zarza-Arizmendi

\section{Artículos de investigación}

Identificación de factores de riesgo para cáncer de próstata

G. L. Ortiz-Arrazola, A. Reyes-Aguilar, I. Grajales-Alonso, I. Tenahua-Quitl

Experiencias y conocimientos de los estudiantes de enfermería, ante la muerte del paciente hospitalizado

M. Á. Orozco-González, G. O. Tello-Sánchez, R. Sierra-Aguillón, R. M. Gallegos-Torres, Á. S. Xeque-Morales, B. L. Reyes-Rocha, A. Zamora-Mendoza

Opinión de los empleadores respecto al desempeño de los egresados del Plan Único de Especialización en Enfermería de la ENEO

M. García-Cardona, R. A. Zárate-Grajales, R. Matus-Miranda, C. L. Balseiro-Almario, C. Balan-Gleaves

Artículos de revisión

Revisión de cavidad uterina instrumentada gentil frente a la revisión manual y su relación con la hemorragia posparto

A. L. Camacho-Villarreal y J. C. Pérez-López

\section{Innovación para la práctica}

Intervenciones de enfermería en una persona con síndrome de Guillain-Barré tipo AMAN, bajo la teoría de Dorothea Orem

K. L. Cancino-Morales, V. Balcázar-Martínez y R. Matus-Miranda

\section{Revista no. 2 Año 10}

\section{Editorial}

ENEO, un logro más: División de Posgrado por fin en Ciudad Universitaria (UNAM)

I. Pérez-Cabrera

\section{Artículos de Investigación}

Depresión y función cognitiva de adultos mayores de una comunidad urbano marginal

T. Durán-Badillo, R. M. Aguilar, M. L. Martínez, T. Rodríguez, G. Gutiérrez y L. Vázquez

Procesos de evaluación del aprendizaje del cuidado en la práctica de estudiantes de Enfermería

M. I. Mantilla-Pastrana y M. C. Gutiérrez-Agudelo 
Artículo de Revisión

El análisis del discurso como perspectiva metodológica para investigadores de salud

E. Urra, A. Muñoz y J. Peñas

Artículos de Innovación para la Práctica

Proceso de atención de Enfermería a pacientes con gangrena de Fournier

S. Mondragón-Gómez y J. Jiménez-Utrilla

Estudio de caso de una preescolar con déficit en los requisitos universales de autocuidado secundario a osteogénesis imperfecta

N. A. Rodríguez-Sandoval, E. Solorzano-García y A. Hernández-Cantoral

\section{Revista no. 3 Año 10}

Editorial

Desarrollo y evolución de la Investigación en Enfermería a través del trabajo en Red. La experiencia de 2 Institutos Nacionales de Salud

M. G. Nava-Galán y S. Hernández-Corral

Artículos de Investigación

Proceso de iniciación de los alumnos de Enfermería, en la formación de las competencias obstétricas

I.Piña-Jiménez

Expresiones y comportamientos de apego madre-recién nacido hospitalizado en Unidad de Cuidado Intensivo Neonatal

M. Guerra-Ramírez y L. Muñoz-de Rodríguez

El tutor clínico. Una mirada de los estudiantes de Licenciatura de Enfermería y Obstetricia

J. L. Hidalgo-Rivera, M. Cárdenas-Jiménez y S. Rodríguez-Jiménez

\section{Artículo de Revisión}

La píldora anticonceptiva a debate

C. González-Hernando, M. Souza-de Almeida, P. Martín-Villamor, M. J. Cao-Torija

y M. J. Castro-Alija

Artículo de Innovación para la Práctica

Proceso de atención de Enfermería, a una adolescente con dependencia en la necesidad de oxigenación por ventrículo único

P. Alvarado-Pacheco y M. Cruz-Jiménez

\section{Revista no. 4 Año 10}

Editorial

Los estudios de doctorado como motor del desarrollo de enfermería

M.C. Müggenburg y Rodríguez Vigil

Artículos de Investigación

Adaptación y validación de un instrumento de valoración de riesgo de caídas

en pacientes pediátricos hospitalizados

J. Barrientos-Sánchez, A. Hernández-Cantoral, M. Hernández-Zavala

Evaluación de competencias de los estudiantes de enfermería a través del Aprendizaje Basado en Problemas

C. González-Hernando, P. Martín-Villamor, M.Á. Carbonero-Martín, F. Lara-Ortega

Alimentación de grupos vulnerables: una mirada a los comedores comunitarios de Tizimín, Yucatán, México

M. Balam-Gómez, G. Uicab-Pool, P. Uch-Puc, J. Sabido-Barrera 
Artículo de Revisión

Las coordenadas pedagógicas del cuidado a los enfermos en las Órdenes Religiosas con Voto de Hospitalidad

J.M. Hernández-Conesa, C. Sellán-Soto, E. Maldonado-Suárez

Artículo de Innovación para la Práctica

Intervenciones de enfermería especializada en un adulto con alteraciones de la marcha ocasionadas por quemadura eléctrica

E.C. Aguilar-Ruíz, N. Ramírez-Gutiérrez, E.C. Camaño-Vicente, S. Hernández-Corral 